\title{
What's Needed for Approval of Fixed-Dose Combination Products?
}

\section{Pierre A Guertin*}

Department of Psychiatry and Neurosciences, Laval University, Quebec City, QC, Canada

\section{Different Types of Combinatorial Approaches}

A combination product is a therapeutic intervention for which more than one therapy or approach is used. This may include therapeutic and diagnostic products that combine drugs, devices, and/ or biological products (e.g. device/drug or biologic/drug products) according to the U.S. Food and Drug Administration (http://www.fda. gov/CombinationProducts/AboutCombinationProducts/default.htm). In the case of drug products per se (i.e., drug-drug combination), they may be composed of several drugs (i.e., small-molecule and/or peptide) marketed as several separate pills, each containing a particular drug (co-packaging), or as single pills that contain several drugs (i.e., fixeddose combination products).

In other words, a fixed-dose combination product (FDC) is a drugdrug combination for which all active moieties (drugs) are assembled with a fixed ratio of doses into one single pill. In some therapeutic areas such as cancer, HIV, malaria, tuberculosis or asthma, they have been used for more than 50 years [1]. Many advantages have thus been associated with FDCs - increased efficacy and/or reduced incidence of adverse effects, lower costs of manufacturing, simpler logistics (distribution), and improved adherence and compliance. For the industry, they constitute generally low-cost and low-risk projects increasing revenues and extending market exclusivity once original patents have expired [2]. From a clinical point of view, FDCs aim either at eliciting additive or synergist effects for the same indication or at inducing new effects for another indication. Examples of products of that first category are numerous. For HIV, asthma, hypertension, cholesterol, several FDCs have become gold-standards and blockbusters therapies. Atripla (efavirenz/emtricitabine/tenofovir disoproxil fumarate), Advair (fluticasone/salmeterol), Janumet (sitagliptine/metformin), Symbicort ${ }^{\circ}$ (budesonide/formoterol), Combivir' (zidovudine/lamivudine), Hyzaar (losartan/HCT), Truvada (emtricitabine/tenofovir) and Vytorin (ezetimibe/simvastatin) have reached combined sales beyond 15 Billion USD annually [3]. One of the newest FDCs to reach market, called Xultophy" (degludec/liraglutide) is believed, according to Novo Nordisk, to become the next gold-standard against type 2 diabetes [4].

\section{Better Effects for Same Indication or New Effects for another Indication}

As mentioned earlier, most FDCs exhibit increased additive or synergistic effects for the same indication(s) the constitutive drugs had been originally approved for (see examples cited above). However, a relatively new type of FDC is emerging - products that can induce brand new effects for the development of new market(s). The FDA considers some of them as new chemical entity (NCE) specifically if one of the active moieties had never been approved. New effects with old drugs may also be considered as NCE with only 3 years of extended market exclusivity. For instance, in spinal cord injury (SCI), a FDC called Spinalon $^{\mathrm{TM}}$ (levodopa/carbidopa/buspirone normally used for Parkinson's disease and anxiety, respectively) was recently found to potently reactivate spinal locomotor neurons and, hence, temporary elicit corresponding episodes of basic walking in chronic paraplegic animals [5]. It has recently completed phase I/II trials (https:// clinicaltrials.gov/ct2/show/NCT01484184). For sleeping disorders, new CNS-mediated actions were found to be induced by THN102 - a FDC comprising an old drug modafinil (in low doses) and a new molecular entity THN02 (currently in phase II clinical development) [6]. Buprenorphine, originally approved as an anesthetic agent (opioid agonist), was recently approved (2014) in combination with naloxone (opioid antagonist) as a novel FDC called Suboxone against opioid dependence [7].

\section{Manufacturing and Bioequivalence Data}

Obviously, if a FDC seeks approval for increased effects or for new effects-corresponding clinical data needs to be provided to regulatory agencies. But beyond that, issues regarding formulation and manufacturing need to be addressed properly $[8,9]$. For instance, when bringing together different APIs, the latter must exhibit physical and chemical compatibility to ensure that the different components do not generate new impurities or lead to drug-drug interactions. If chemically incompatible APIs are found, it is imperative to develop a multi-layer approach such as with an intermediate placebo layer to avoid interactions. Otherwise, mainly bioequivalence data in healthy individuals (phase I with 20-30 subjects) are expected by authorities for NDA approval to demonstrate that the each API maintains its PK/PD profile. For FDCs that propose new effects (using only old drugs), standard clinical data (phase II-III trials) are required although the phase I study in health subjects is not generally required since compelling safety data normally exist for each API $[9,10]$.

\section{Conclusion}

The development of new FDCs is increasingly popular. In neurology, where innovative products are lacking, this may become a novel opportunity for the industry to ease and accelerate the development of new products [11].

\section{References}

1. Wofford JL (1997) History of fixed-dose combination therapy for hypertension Arch Intern Med 157: 1044

2. Hao J, Rodriguez-Monguio R, Seone-Vazquez E (2016) Fixed-dose combination drug approvals, patents and market exclusivities compared to single active ingredient pharmaceuticals. PLoS One 10: 1-13.

3. Pourkavoos N (2012) Unique risks, benefits, and challenges of developing drug-drug combination products in a pharmaceutical industrial setting. Comb Prod Ther 2: 1-31.

4. Kararli TT (2011) Fixed dose combination products overview. Presentation of Pharma Circle LLC.

*Corresponding author: Dr. Pierre A Guertin, Professor, Department of Psychiatry and Neurosciences, Laval University and Laval University Medical Center (CHU de Québec) 2705 Laurier Boulevard, RC-9800 (Neuroscience Unit), Quebec City, QC, G1Y 2T4, Canada, Tel: +14185254444; Fax: +14186542753; E-mail: pierre.guertin@crchul.ulaval.ca

Received April 27, 2016; Accepted April 29, 2016; Published May 05, 2016

Citation: Guertin PA (2016) What's Needed for Approval of Fixed-Dose Combination Products? J Bioequiv Availab 8: e71. doi:10.4172/jbb.10000e71

Copyright: () 2016 Guertin PA. This is an open-access article distributed under the terms of the Creative Commons Attribution License, which permits unrestricted use, distribution, and reproduction in any medium, provided the original author and source are credited. 
5. http://www.prnewswire.com/news-releases/novo-nordisks-insulindegludecliraglutide-is-set-to-become-the-clinical-gold-standard-among-fixeddose-combination-therapies-for-type-2-diabetes-266037201.html

6. Guertin PA, Ung RV, Rouleau P (2010) Oral administration of a tri-therapy for central pattern generator activation in paraplegic mice: proof-of-concept of efficacy. Biotechnol J 5: 421-426.

7. Mouthon F, Charveriat M, Deslys JP, Iris F. Use of anti-connexin agents for modulating the therapeutic effect of psychotropic drugs. US patent 14/736,004.

8. Van Doren BA, Foulks-Rodriguez KA, Yarborough W (2016) Opioid addiction treatment using buprenorphine-naloxone in a community-based internal medicine practice. J Okla State Med Assoc 108: 303-309.

9. (2006) US Department of Health and Human Services. Guidance for industryfixed-dose combinations, co-packaged drug products, and single-entity version of previously approved antiretrovirals for the treatment of HIV. Procedural.

10. World Health Organization (2005) WHO Expert Committee on Specifications for Pharmaceutical Preparations. World Health Organ Tech Rep Ser 929: 1-142, back cover.

11. Wegener G, Rujescu D (2013) The current development of CNS drug research Int J Neuropsychopharmacol 16: 1687-1693. 